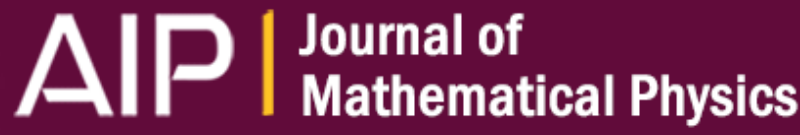

\section{Area law for fixed points of rapidly mixing dissipative quantum systems}

Fernando G. S. L. Brandão, Toby S. Cubitt, Angelo Lucia, Spyridon Michalakis, and David PerezGarcia

Citation: Journal of Mathematical Physics 56, 102202 (2015); doi: 10.1063/1.4932612

View online: http://dx.doi.org/10.1063/1.4932612

View Table of Contents: http://scitation.aip.org/content/aip/journal/jmp/56/10?ver=pdfcov

Published by the AIP Publishing

\section{Articles you may be interested in}

Quantum logarithmic Sobolev inequalities and rapid mixing

J. Math. Phys. 54, 052202 (2013); 10.1063/1.4804995

Hartman effect and dissipative quantum systems

J. Math. Phys. 54, 052101 (2013); 10.1063/1.4803132

Characterizations of fixed points of quantum operations

J. Math. Phys. 52, 052103 (2011); 10.1063/1.3583541

Fixed points of quantum operations

J. Math. Phys. 43, 5872 (2002); 10.1063/1.1519669

Quantum dissipative systems

AIP Conf. Proc. 438, 1 (1998); 10.1063/1.56342

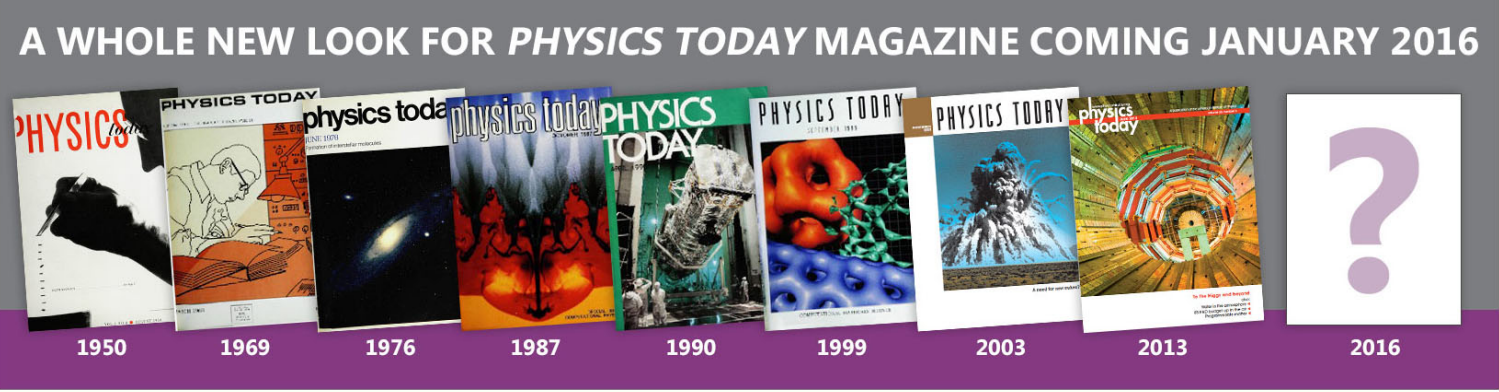




\title{
Area law for fixed points of rapidly mixing dissipative quantum systems
}

\author{
Fernando G. S. L. Brandão, ${ }^{1,2}$ Toby S. Cubitt, ${ }^{2,3}$ Angelo Lucia, ${ }^{4, a)}$ \\ Spyridon Michalakis, ${ }^{5}$ and David Perez-Garcia ${ }^{4,6,7}$ \\ ${ }^{1}$ Quantum Architectures and Computation Group, Microsoft Research, Redmond, \\ Washington 98052, USA \\ ${ }^{2}$ Department of Computer Science, University College London, Gower Street, \\ London WCIE 6BT, United Kingdom \\ ${ }^{3}$ DAMTP, University of Cambridge, Cambridge, United Kingdom \\ ${ }^{4}$ Departamento de Análisis Matemático, Universidad Complutense de Madrid, \\ Madrid, Spain \\ ${ }^{5}$ Institute for Quantum Information and Matter, Caltech, California 91125, USA \\ ${ }^{6}$ IMI, Universidad Complutense de Madrid, Madrid, Spain \\ ${ }^{7}$ ICMAT, C/Nicolás Cabrera, Campus de Cantoblanco, 28049 Madrid, Spain
}

(Received 22 June 2015; accepted 26 September 2015; published online 13 October 2015)

\begin{abstract}
We prove an area law with a logarithmic correction for the mutual information for fixed points of local dissipative quantum system satisfying a rapid mixing condition, under either of the following assumptions: the fixed point is pure or the system is frustration free. (C) 2015 AIP Publishing LLC. [http://dx.doi.org/10.1063/1.4932612]
\end{abstract}

\section{INTRODUCTION}

One of the problems common to quantum information and condensed matter physics is understanding correlations and entanglement in many-body quantum states. This is motivated by the observation that many interesting states behave very differently from random states: while in the latter, the entanglement entropy of a sub-region scales as the volume of the region, and in the states that occur in quantum many-body systems, this entanglement entropy is often seen to scale only with the boundary of the region. This surprising behaviour has been called the area law (the terminology comes from 3D systems), and it is a well-studied conjecture that ground states of (gapped) local Hamiltonians should satisfy an area law. Even in the case of critical systems and gapless Hamiltonians, evidence suggests that the ground state still has a sub-volume growth of the entanglement, with a rate proportional to the boundary of the region times a logarithmic correction. ${ }^{1-3}$ While it has been formally proven only for 1D systems, ${ }^{4-8}$ area laws have also been proven in specific cases in higher dimensions (harmonic lattice systems, ${ }^{9}$ models satisfying local topological quantum order, ${ }^{10}$ perturbations of gapped Hamiltonians satisfying an area law, ${ }^{11,12}$ and with a logarithmic correction for fermionic systems ${ }^{13,14}$ ) and are the subject of active research.

Recently, a different class of states arising in quantum many-body systems has been attracting attention in the quantum information literature: fixed points of (local) dissipative processes. More precisely, fixed points of semigroups of trace preserving completely positive linear maps. The motivation is two-fold: on the one hand, such processes model most of the different types of noise that can be found in nature and therefore provide a more realistic model for physical systems, since in practice no system will be completely isolated. On the other hand, proposals have been made to artificially engineer such dissipative interactions in order to have a determined quantum state as a fixed point, effectively making them "dissipative machines" for producing useful/interesting quantum states. ${ }^{15,16}$ This dissipative state engineering has been experimentally shown to be a robust mechanism to maintain coherence. ${ }^{17,18}$

\footnotetext{
a)Electronic mail: anlucia@ucm.es
} 
A natural question then arises: is there an area law (either strict or with a logarithmic correction) in this context? Note that since fixed points of dissipative evolutions are generically not pure, we must find another measure of entanglement or correlations, since local entropy is no longer a useful measure for mixed states (as the trivial example of the maximally mixed state shows). ${ }^{19}$ proposed instead using the mutual information, a measure of correlations between two parts of a quantum state. It has the advantage of coinciding with entanglement entropy for pure states, and it upper bounds operational measures of entanglement in the mixed-state case, such as the distillable entanglement. ${ }^{20}$ In Ref. 19, it was also shown that thermal states of local Hamiltonians satisfy an area law for the mutual information. However, thermal states do not cover all the possible fixed points of dissipative systems. Pinning down under which conditions an area law for the mutual information holds for general dissipative quantum many-body systems is thus an interesting open problem.

For Hamiltonian systems, the main assumption that is usually made is the presence of a spectral gap: a non-vanishing separation between the two lowest energy levels of the Hamiltonian. In the dissipative setting, instead of spectral assumptions, it is more natural to make assumptions on the speed of convergence of the dissipation towards its fixed point (a quantity that is not controlled by the spectrum alone ${ }^{21}$ ) or equivalently on the so-called mixing time. In this work, we restrict to systems for which the mixing time scales logarithmically with the system size. In a previous paper, ${ }^{22}$ some of the authors showed that such systems - which we called rapid mixing - are stable under local perturbations. Similarly to the gap of closed quantum systems, proving rapid mixing for dissipative systems is a daunting task. There are however some interesting key examples: state preparation of graph states, ${ }^{23}$ classical Glauber dynamics for the Ising model in 2D (in some range of parameters), ${ }^{24,25}$ among others.

Rapid mixing is implied in many cases by a well studied property of dissipative evolutions, namely, the existence of a system-size independent Log-Sobolev constant for primitive reversible Liouvillians. ${ }^{26-30}$ Under such assumptions, in Ref. 31, it was proven a bound on the mutual information of the form:

$$
I\left(A: A^{c}\right) \leq c \log \log \left\|\rho^{-1}\right\||\partial A| .
$$

In the latter bound, $\rho$ is the fixed point of the evolution, and therefore, $\left\|\rho^{-1}\right\|$ will usually depend at least exponentially on the total system size, and sometimes even worse. As recognized by the authors of Ref. 31, this poses a serious problem in considering Eq. (1) a satisfactory area law. It indicates however that rapid mixing seems to be the required condition to have an area law in the dissipative setting. This is exactly what we prove in the present paper, with the following results:

1. if the system satisfies rapid mixing and the fixed point is pure, then it satisfies an area law with a logarithmic correction for the entanglement entropy;

2. if the system satisfies rapid mixing and is frustration free, meaning that the local terms of the Liouvillian share a common steady state, then such fixed point satisfies an area law with a logarithmic correction for the mutual information.

Compared with (1), the bounds we obtain do not have any dependence on the total system size. Moreover, we do not require primitivity or reversibility of the generators of the evolution, and we only require rapid mixing instead of a system-size independent Log-Sobolev constant (a strictly weaker assumption, since the Log-Sobolev constant is undefined for non-primitive Liouvillians).

It is known that there is a connection between area laws and decay of correlations. ${ }^{7,19}$ Therefore, it does not come as a surprise that with the tools we have developed for proving the area law, we can also prove a decay of correlations measured with the mutual information. It is worth noting that with the results available in the literature and to the best of our knowledge, it is not possible to derive the area law from the type of decay of correlations we will show here.

As we have mentioned earlier, for ground states of closed systems, a logarithmic correction is usually considered a signature of a gapless Hamiltonian. For open systems and mixed states, the situation is less clear: already in Ref. 19, it was shown that thermal states of local Hamiltonian satisfy an area law without a logarithmic correction irrespective of the gap of the Hamiltonian. For these states, the bound we obtained is therefore not optimal. We do not know whether there exist 
systems which saturate our bound or if instead the correction is only an artefact of the proof. If there exist systems that saturate our bound, it would then imply that their fixed point have a very interesting property: while still satisfying an exponential decay of correlations, they do not satisfy an area law without a logarithmic correction. Having an example of such state which can also be efficiently prepared with a dissipative process would be interesting on its own, as it could lead to new insight into the relationship between area laws and decay of correlations.

We conjecture that rapid mixing alone, without any additional assumptions, should imply an area law for mutual information, but we do not have a formal proof. The fact that we have two different proofs of an area law, requiring different extra assumptions (pure fixed point on the one hand, frustration freeness on the other) is strong evidence for this conjecture.

The paper is organized as follows. In Section II, we set up the problem and introduce the necessary notation and definitions. In Section III, we prove two lemmas regarding localization properties of the fixed point of the dissipative maps: Lemma 11 is based only on the rapid mixing assumption and will be used in Section IV to prove decay of correlations and in Section V to prove the area law for pure fixed points; Lemma 12 instead requires the extra assumption of frustration freeness and will be used in Section $V$ to prove the area law for the mutual information in the case of mixed fixed points.

\section{SETUP AND NOTATION}

Let $\mathcal{H}_{A B}=\mathcal{H}_{A} \otimes \mathcal{H}_{B}$ be a finite dimensional complex Hilbert space, representing a bipartite quantum system. We denote by $\mathcal{B}\left(\mathcal{H}_{A B}\right)$ the space of bounded linear operators on $\mathcal{H}_{A B}$. A state is given by a positive semi-definite operator $\rho_{A B} \in \mathcal{B}\left(\mathcal{H}_{A B}\right)$, normalized to have trace equal to one. The reduced density matrix of the subsystem A (respectively, B) will be denoted by $\rho_{A}$ (respectively, $\rho_{B}$ ), and it is given by $\rho_{A}=\operatorname{Tr}_{A} \rho_{A B}$ (respectively, $\rho_{B}=\operatorname{Tr}_{B} \rho_{A B}$ ), where the partial trace $\operatorname{Tr}_{A}$ is defined to be the unique linear operator $\operatorname{Tr}_{A}: \mathcal{B}\left(\mathcal{H}_{A B}\right) \rightarrow \mathcal{B}\left(\mathcal{H}_{B}\right)$ such that $\operatorname{Tr}_{A}(x \otimes y)=y \operatorname{Tr}(x)$ for all $x$ in $\mathcal{B}\left(\mathcal{H}_{A}\right)$ and all $y$ in $\mathcal{B}\left(\mathcal{H}_{B}\right)\left(\operatorname{Tr}_{B}\right.$ is similarly defined).

We will use the standard Dirac notation for Hilbert spaces, denoting vectors as $|\phi\rangle$, adjoint vectors as $\langle\phi|,\langle\phi \mid \psi\rangle$ for the scalar product, and $|\phi\rangle\langle\psi|$ for rank one linear maps. The canonical basis will be indexed by natural numbers starting from zero: $|0\rangle,|1\rangle, \ldots,|n\rangle$.

We will denote by $\operatorname{poly}(x)$ any polynomial in the variable $x$ with real coefficients and arbitrary degree.

\section{A. Measures of correlations}

Given a state $\rho_{A B} \in \mathcal{B}\left(\mathcal{H}_{A B}\right)$ of a bipartite system, there are a number of possible measures of how "distant" the state $\rho_{A B}$ is from being a product state, i.e., of the form $x \otimes y$ for some $x$ in $\mathcal{B}\left(\mathcal{H}_{A}\right)$ and $y$ in $\mathcal{B}\left(\mathcal{H}_{B}\right)$. Since a product state represents a system in which measurements over the subsystem $A$ are independent of measurements over the subsystem $B$, we will talk of correlation measures between subsystems $A$ and $B$. We will need to define and use three of such measures. We will follow the same terminology of Ref. 31 .

\section{Definition 1 (Correlation measures).}

- Covariance correlation:

$$
\begin{aligned}
C(A: B) & =\max _{\substack{M \in \mathcal{B}\left(\mathcal{H}_{A}\right), N \in \mathcal{B}\left(\mathcal{H}_{B}\right) \\
\|\boldsymbol{M}\| \leq 1,\|N\| \leq 1}}|\langle M \otimes N\rangle-\langle M\rangle\langle N\rangle| \\
& =\max _{\substack{M \in \mathcal{B}\left(\mathcal{H}_{A}\right), N \in \mathcal{B}\left(\mathcal{H}_{B}\right) \\
\|M\| \leq 1,\|N\| \leq 1}}\left|\operatorname{Tr}\left[M \otimes N\left(\rho_{A B}-\rho_{A} \otimes \rho_{B}\right)\right]\right|,
\end{aligned}
$$

where $\langle O\rangle=\operatorname{Tr}\left(O \rho_{A B}\right)$ is the expectation value of the observable $O$ acting on $\rho_{A B}$. 
- Trace distance correlation:

$$
\begin{aligned}
T(A: B) & =\max _{\substack{F \in \mathcal{B}\left(\mathcal{H}_{A B}\right) \\
\|F\| \leq 1}}\left|\operatorname{Tr}\left[F\left(\rho_{A B}-\rho_{A} \otimes \rho_{B}\right)\right]\right| \\
& =\left\|\rho_{A B}-\rho_{A} \otimes \rho_{B}\right\|_{1} .
\end{aligned}
$$

- Mutual information correlation:

$$
I(A: B)=S\left(\rho_{A}\right)+S\left(\rho_{B}\right)-S\left(\rho_{A B}\right),
$$

where $S(\rho)=-\operatorname{Tr}\left(\rho \log _{2} \rho\right)$ is the von Neumann entropy of the state $\rho$.

When it is not clear from context which state $\rho_{A B}$ we are considering, we indicate it in a subscript to avoid ambiguity and write $C(A: B)_{\rho}, T(A: B)_{\rho}$, and $I(A: B)_{\rho}$.

As it should be clear from the definition, $C(A: B)$ is always upper bounded by $T(A: B)$. Moreover, by Pinsker's inequality, ${ }^{32}$

$$
C(A: B) \leq T(A: B) \leq 2 \sqrt{I(A: B)} .
$$

Therefore, mutual information is the strongest correlation measure. It is also a well known consequence of the Alicki-Fannes-Audenaert inequalities ${ }^{33-35}$ that there is a non-linear inverse relationship between trace distance and mutual information: there is a differentiable function $f(x)$ vanishing at zero such that $I(A: B) \leq f(T(A: B))$. Such non-linear equivalence between the two measures will allow us to take bounds on $T(A: B)$ (which in the context of our assumptions will be easier to deduce) and obtain information on the behavior of $I(A: B)$.

We state this result in a form that will be more convenient for us and, for the sake of completeness, present a short proof of it.

For $x \in[0,1], h_{b}(x)=-x \log _{2} x-(1-x) \log _{2}(1-x)$ denotes the binary entropy function.

Theorem 2. The following inequalities hold:

Fannes-Audenaert: ${ }^{33,34}$

Let $\rho, \sigma \in \mathcal{B}\left(\mathbb{C}^{d}\right)$, and let $\delta=\|\rho-\sigma\|_{1}<1$. Then,

$$
|S(\rho)-S(\sigma)| \leq 2 \delta \log _{2}(d-1)+2 h_{b}(\delta) .
$$

\section{Alicki-Fannes: ${ }^{35}$}

Let $\rho^{A B}, \sigma^{A B} \in \mathcal{B}\left(\mathbb{C}^{d_{A}} \otimes \mathbb{C}^{d} B\right)$, and let $\delta=\left\|\rho^{A B}-\sigma^{A B}\right\|_{1}<1$. Then,

$$
\left|S\left(\rho^{A B} \mid \rho^{B}\right)-S\left(\sigma^{A B} \mid \sigma^{B}\right)\right| \leq 4 \delta \log _{2} d_{A}+2 h_{b}(\delta),
$$

where the conditional von Neumann entropy $S\left(\rho^{A B} \mid \rho^{B}\right)$ is defined as $S\left(\rho^{A B} \mid \rho^{B}\right)=S\left(\rho^{A B}\right)-$ $S\left(\rho^{B}\right)$.

Combining the two previous inequalities, we will obtain the desired non-linear bound on $I(A: B)$.

Corollary 3. Let $\delta=\left\|\rho^{A B}-\sigma^{A B}\right\|_{1}<1$. Then,

$$
\left|I(A: B)_{\rho}-I(A: B)_{\sigma}\right| \leq 6 \delta \log _{2} d_{A}+4 h_{b}(\delta) .
$$

In particular, if we take $\sigma^{A B}=\rho^{A} \otimes \rho^{B}$, we have that $I(A: B)_{\sigma}=0, \delta=T(A: B)_{\rho}$, and thus,

$$
I(A: B)_{\rho} \leq 6 T(A: B)_{\rho} \log _{2} d_{A}+4 h_{b}\left(T(A: B)_{\rho}\right) .
$$

Proof. Note that $\left\|\rho^{A B}-\sigma^{A B}\right\|_{1} \leq \delta \Rightarrow\left\|\rho^{A}-\sigma^{A}\right\|_{1} \leq \delta$. Applying triangle inequality and Equations (2) and (3) gives

$$
\left|I(A: B)_{\rho}-I(A: B)_{\sigma}\right| \leq\left|S\left(\rho^{A}\right)-S\left(\sigma^{A}\right)\right|+\left|S\left(\rho^{A B} \mid \rho^{B}\right)-S\left(\sigma^{A B} \mid \sigma^{B}\right)\right| .
$$




\section{B. Many-body quantum systems}

Let us now recall the standard definitions and the common notation for many-body quantum systems.

We will consider a quantum system defined on the square lattice $\Gamma=\mathbb{Z}^{D}$ equipped with the graph metric, where at each site $x \in \Gamma$, we associate a finite-dimensional complex Hilbert space $\mathcal{H}_{x}$. We choose to work with a square lattice for simplicity of exposition, but the results presented can be generalized straightforwardly to graphs with polynomial growth (i.e., with balls size growing polynomially with the diameter). The ball centered at $x$ of radius $r$ will be denoted by $b_{x}(r)$. We will use the following convention: given a subset $A \subset \Lambda$, we will denote by $A(s)$ the smallest disjoint union of balls containing $\{x \in \Lambda \mid \operatorname{dist}(x, A) \leq s\}$. For each finite subset $\Lambda \subset \Gamma$ of the lattice, we will associate a Hilbert space $\mathcal{H}_{\Lambda}=\otimes_{x \in \Lambda} \mathcal{H}_{x}$ and an algebra of observables $\mathcal{A}_{\Lambda}=\mathcal{B}\left(\mathcal{H}_{\Lambda}\right)$. We will equip $\mathcal{A}_{\Lambda}$ with the Hilbert-Schmidt scalar product $\langle A, B\rangle=\operatorname{Tr}\left(A^{*} B\right)$.

A linear map $\mathcal{T}: \mathcal{A}_{\Lambda} \rightarrow \mathcal{A}_{\Lambda}$ will be called a superoperator ${ }^{36}$ to stress the fact that is an operator acting on operators. Its support is defined to be the minimal set $\Lambda^{\prime} \subseteq \Lambda$ such that $\mathcal{T}=\mathcal{T}^{\prime} \otimes \mathbb{1}$, where $\mathcal{T}^{\prime} \in \mathcal{B}\left(\mathcal{A}_{\Lambda^{\prime}}\right)$. Positivity is defined as usual for linear maps: $\mathcal{T}$ is said to be positive if it maps positive operators to positive operators. $\mathcal{T}$ is called completely positive if $\mathcal{T} \otimes \mathbb{1}: \mathcal{A}_{\Lambda} \otimes M_{n} \rightarrow \mathcal{A}_{\Lambda} \otimes M_{n}$ is positive for all $n \geq 1$. Finally, we say that $\mathcal{T}$ is trace preserving if $\operatorname{Tr} \mathcal{T}(\rho)=\operatorname{Tr} \rho$ for all $\rho \in A_{\Lambda}$.

A dissipative evolution for a quantum system is given by a one-parameter continuous semigroup of completely positive and trace preserving superoperators $\left\{T_{t}: \mathcal{A}_{\Lambda} \rightarrow \mathcal{A}_{\Lambda}\right\}_{t}$. If $\rho \in \mathcal{B}\left(\mathcal{H}_{\Lambda}\right)$ is the state of the system at time zero, then the evolution of $\rho$ at time $t \geq 0$ is given by $\rho(t)=T_{t}(\rho)$. The assumptions on $T_{t}$ guarantee that $\rho(t)$ is again a state, i.e., a positive and trace one operator. This is usually called the Schrödinger picture.

We will make use of the following norm for superoperators:

$$
\|T\|_{\diamond}=\sup _{n}\left\|T \otimes \mathbb{1}_{n}\right\|_{1 \rightarrow 1}=\sup _{n} \sup _{\substack{X \in \mathcal{A}_{\Lambda} \otimes M_{n} \\ X \neq 0}} \frac{\left\|T \otimes \mathbb{1}_{n}(X)\right\|_{1}}{\|X\|_{1}} .
$$

Dissipative maps are contractive with respect to such norm, in the sense that $\|\mathcal{T}\|_{\diamond} \leq 1$.

Given a semigroup of dissipative maps $\left\{T_{t}\right\}$, it has a generator $\mathcal{L}: \mathcal{A}_{\Lambda} \rightarrow \mathcal{A}_{\Lambda}$ which satisfies $\frac{\mathrm{d}}{\mathrm{d} t} T_{t}(\rho)=\mathcal{L} T_{t}(\rho)$. Such superoperator is called a Lindbladian or Liouvillian (we will use the former). The assumptions made on $T_{t}$ force a particular structure on $\mathcal{L}$, which is called the Lindblad form. ${ }^{37,38}$ A superoperator $\mathcal{L}$ is said to be in the Lindblad form if it can be written as

$$
\mathcal{L}(\rho)=i[\rho, H]+\sum_{j} L_{j} \rho L_{j}^{*}-\frac{1}{2}\left\{L_{j}^{*} L_{j}, \rho\right\},
$$

where $H$ is a Hermitian operator, $\left(L_{j}\right)_{j}$ are arbitrary operators (called the Lindblad operators), $[\cdot, \cdot]$ denotes the commutator, and $\{\cdot, \cdot\}$ denotes the anti-commutator. We refer to Refs. 36 and 39 for details on the theory of Lindblad operators.

As shown in Ref. 36, given a semigroup of dissipative maps $\left\{T_{t}\right\}_{t}$, we can define a new map $T_{\infty}$ representing the "infinite time limit" of the evolution, or in other words, the projector onto the space of fixed points of the evolution. $T_{\infty}$ is again a completely positive, trace preserving superoperator, since it can be obtained as $\lim _{N \rightarrow \infty} \frac{1}{N} \sum_{n=0}^{N} T_{n}$.

\section{Uniform families}

Given a generator $\mathcal{L}$, we can decompose it as a sum of local terms, i.e., terms which are still of the Lindblad form but with controlled support,

$$
\mathcal{L}=\sum_{Z \subset \Lambda} \mathcal{L}_{Z}, \quad \operatorname{supp} \mathcal{L}_{Z}=Z, \quad \mathcal{L}_{Z} \text { is Lindbladian. }
$$

When $\left\|\mathcal{L}_{Z}\right\|_{\diamond}$ is decaying with $\operatorname{diam} Z$, we will generically say that the evolution is local. More stringent assumptions on the decay rate of the norms of the local generators will be required and are formalized in Assumptions (A1) and (A2) in this section. 
Since we are interested in dissipative evolutions defined on increasing sequences of lattices and how their properties depend on the lattice size (often referred to as the system size), we need to define a meaningful way of growing the evolutions with the lattice size, adding, and modifying the necessary generator terms appropriately. As presented in Ref. 22, the following definition of uniform families of dissipative evolutions is one solution to this, which is general enough to cover a wide range of models and situations.

Definition 4. Given $\Lambda \subset \Gamma$, a boundary condition for $\Lambda$ is given by a Lindbladian $\mathcal{B}^{\partial \Lambda}=$ $\sum_{d \geq 1} \mathcal{B}_{d}^{\partial \Lambda}$, where $\operatorname{supp} B_{d}^{\partial \Lambda} \subset \partial_{d} \Lambda:=\left\{x \in \Lambda \mid \operatorname{dist}\left(x, \Lambda^{c}\right) \leq d\right\}$.

Definition 5. A uniform family of Lindbladians is given by the following:

(i) infinite Lindbladian: a local Lindbladian $\mathcal{M}$ defined all of $\mathbb{Z}^{D}: \mathcal{M}=\sum_{Z \subset \mathbb{Z}^{D}} \mathcal{M}_{Z}$;

(ii) boundary conditions: a family of boundary conditions $\left\{\mathcal{B}^{\partial \Lambda}\right\}_{\Lambda}$, where $\Lambda=b_{u}(L)$, for each $u \in \mathbb{Z}^{D}$ and $L \geq 0$.

Definition 6. A local Lindbladian $\mathcal{L}=\sum_{Z \subset \mathbb{Z}^{D}} \mathcal{L}_{Z}$ is said to be translationally invariant if $\mathcal{L}_{Z+u}=\mathcal{L}_{Z}, \forall u \in \mathbb{Z}^{D}$.

We say that a uniform family $\mathcal{L}=\{\mathcal{M}, \mathcal{B}\}$ is translationally invariant if $\mathcal{M}$ is translationally invariant, and moreover, $\mathcal{B}^{\partial b_{u}(L)}$ is independent of $u$.

Given a uniform family $\mathcal{L}=\{\mathcal{M}, \mathcal{B}\}$, we fix the following notation for evolutions defined on $\Lambda=b_{u}(L) \subset \Gamma$ :

$$
\begin{aligned}
\mathcal{L}^{\Lambda} & =\sum_{Z \subset \Lambda} \mathcal{M}_{Z} \quad \text { "open boundary” evolution, } \\
\mathcal{L}^{\bar{\Lambda}}=\mathcal{L}^{\Lambda}+\mathcal{L}^{\partial \Lambda} & \text { "closed boundary" evolution, }
\end{aligned}
$$

with the respective evolutions $T_{t}^{\Lambda}=\exp \left(t \mathcal{L}^{\Lambda}\right)$ and $T_{t}^{\bar{\Lambda}}=\exp \left(t \mathcal{L}^{\bar{\Lambda}}\right)$.

Until now, we have made no specific assumption on the decay rate of the norms of the local generators. As mentioned above, in order to meaningfully talk about locality of the evolution, we need to impose that $\left\|\mathcal{L}_{Z}\right\|_{\diamond}$ is decaying with diam $Z$. The rate at which such function decays classifies the system into one of the specific cases more usually considered in the literature: compactly supported (usually called finite-range interactions), exponentially decaying, super-polynomially decaying, power-law decaying, etc. We will take a more general approach and will simply assume from now on that our family of Liouvillians satisfies the following assumptions.

Definition 7 (Lieb-Robinson assumptions). There exists an increasing function $v(r)$ satisfying $v(x+y) \leq v(x) v(y)$, such that the following conditions hold:

$$
\begin{gathered}
\sup _{x \in \Gamma} \sum_{Z \ni x}\left\|\mathcal{M}_{Z}\right\|_{\diamond}|Z| v(\operatorname{diam} Z) \leq v<\infty, \\
\sup _{x \in \Gamma} \sup _{r} v(r) \sum_{d=r}^{N}\left\|\mathcal{B}_{d}^{\partial B(x, N)}\right\|_{\diamond} \leq \operatorname{poly}(N) .
\end{gathered}
$$

If $\left\|\mathcal{M}_{Z}\right\|_{\diamond}$ is exponentially decaying or is compactly supported, then one can take $v(r)=$ $\exp (\mu r)$ for some positive $\mu$. On the other hand, if $\left\|\mathcal{M}_{Z}\right\|_{\diamond}$ decays only polynomially, then we must take $v(r)=(1+r)^{\mu}$. In the latter case, the Lieb-Robinson bounds only hold if $\mu$ is bigger than a constant depending on the geometrical dimension of the lattice $\Gamma$, i.e., $D$ if $\Gamma=\mathbb{Z}^{D}$. The details of when we can apply Lieb-Robinson bounds in this case can be found in our previous work. ${ }^{22}$ From now on, we will simply assume that $v(\cdot)$ decays sufficiently fast for the Lieb-Robinson bounds to apply. 


\section{Frustration freeness}

The following definition is inspired by the analogous concept defined for closed systems and Hamiltonian dynamics. It captures the idea that a fixed point of a local evolution might or might not be locally steady. The local dissipative terms could in general have a non-trivial action on the fixed point, so that it is only the sum of such local effects that adds up to zero and leaves the state invariant. Assuming that the system is frustration free means excluding such cases.

Definition 8. We say that a uniform family $\mathcal{L}=\{\mathcal{M}, \mathcal{B}\}$ satisfies frustration freeness (or is frustration free) iffor all $\Lambda$ and all fixed points $\rho_{\infty}$ of $T_{t}^{\bar{\Lambda}}$,

$$
\mathcal{M}_{Z}\left(\rho_{\infty}\right)=0 \quad \forall Z \subset \Lambda .
$$

$$
T_{t}^{\Lambda}
$$

Remark 9. If $\mathcal{L}=\{\mathcal{M}, \mathcal{B}\}$ is frustration free, then each fixed point of $T_{t}^{\bar{\Lambda}}$ is also a fixed point of

While not true in general, the frustration freeness condition is satisfied by a large class of interesting dissipative systems, as the following examples show:

1. dissipative state engineering procedures defined in Refs. 15 and 16;

2. locally reversible classical Markov chains;

3. locally detailed balanced quantum Markov processes and in particular, Gibbs samplers for commuting Hamiltonians. ${ }^{40}$

\section{LOCALIZATION RESULTS}

A well known property of many-body systems with local interactions, either dissipative or Hamiltonian, is the existence of a finite speed of propagation. This describes how the support of a localized observable spreads in time during the evolution: up to an exponentially small correction, the support spreads linearly with time. The finite velocity at which such linear growth occurs is often called a Lieb-Robinson velocity or sometimes a group velocity. (It is a property of the model and not a consequence of some relativistic effect - we are considering only non-relativistic models here.) While the original work focused on Hamiltonian systems and groups of automorphisms, ${ }^{41,42}$ the existence of such finite speed of propagation in the lattice has been generalized to dissipative evolutions, ${ }^{43,44}$ and in Ref. 22, we showed that the definition of boundary condition we have given allows us to recover the same type of localization properties of the evolution.

Nonetheless, all the Lieb-Robinson localization bounds have a time-dependency, becoming worse as time increases, until the bound they provide becomes trivial and does not give any information at all about the properties of the fixed point. In Secs. III A and III B, we want to produce results which might be interpreted as "infinite time" versions of Lieb-Robinson bounds. To do so, we will need to make an extra assumption on the evolution: we will assume that the convergence to the fixed point is fast, in the sense that scales logarithmically with the system size. This is formalized in the definition of rapid mixing below. Such a definition can be in some cases relaxed to allow convergence which is only scaling sub-linearly with respect to system size. (We will not pursue such generalizations here and instead refer to Ref. 22 for guidance on which changes are necessary to the results below.)

The localization lemma that we prove, Lemma 11, will be only sufficient to prove an area law for the pure fixed point case not for the mutual information. Therefore, we also prove a stronger result, Lemma 12, for which we will need to add the extra hypothesis of frustration freeness.

\section{A. Rapid mixing}

In this section, we want to briefly recall a result proven in Ref. 22 . We start by recalling the definition of rapid mixing. 
Definition 10 (Rapid mixing). Let $\left\{T_{t}^{\Lambda}\right\}_{\Lambda}$ be a family of dissipative maps, we say it satisfies rapid mixing if there exist $c, \gamma, \delta>0$ such that

$$
\sup _{\substack{\rho \geq 0 \\ \operatorname{Tr} \rho=1}}\left\|T_{t}^{\Lambda}(\rho)-T_{\infty}^{\Lambda}(\rho)\right\|_{1} \leq c|\Lambda|^{\delta} e^{-t \gamma} .
$$

In analogy to the spectral gap for Hamiltonians, proving that a family of Lindbladians is rapid mixing is not an easy task. Nonetheless, there exists a large class of interesting systems for which we already have mixing time estimates that imply rapid mixing.

1. (Trivially) non-interacting particle systems.

2. Dissipative state engineering for graph states. ${ }^{23}$

3. Quantum and classical Markov processes satisfying a Log-Sobolev inequality. ${ }^{29}$ This includes in particular Glauber dynamics for the Ising model in 2D, either above the critical temperature or with non-zero magnetic field. ${ }^{24}$

Lemma 11. Let $\mathcal{L}=\{\mathcal{M}, \mathcal{B}\}$ be a uniform family of dissipative evolutions that satisfies rapid mixing, and suppose each $T_{t}^{\bar{\Lambda}}$ has a unique fixed point and no other periodic points. Fix a $\Lambda$ and let $\rho_{\infty}$ be the unique fixed point of $T_{t}^{\bar{\Lambda}}$. Given $A \subset \Lambda$, for each $s \geq 0$ denote by $\rho_{\infty}^{s}$ the unique fixed point of $T_{t}^{\bar{A}(s)}$.

Then, we have

$$
\left\|\operatorname{Tr}_{A^{c}}\left(\rho_{\infty}-\rho_{\infty}^{s}\right)\right\|_{1} \leq|A|^{\delta} \Delta_{0}(s),
$$

for some fast-decaying function $\Delta_{0}(s)$ and some positive constant $\delta$.

The decay rate of $\Delta_{0}(s)$ is in the same class as $v^{-1}(s)$, where $v(s)$ is defined by Assumptions (A1) and (A2): it is exponential if $v^{-1}(s)$ is exponential and polynomial if $v^{-1}(s)$ is polynomial. In the latter case, the degree of the polynomial controlling the decay is smaller than that of $v^{-1}(s)$, but the loss is independent of the system size - again, this corresponds to requiring a sufficiently fast-decaying $v^{-1}(s)$.

\section{B. Localizing with frustration freeness}

In Sec. III B, we want to show a property of the fixed points of a uniform family of Lindbladians verifying frustration freeness. We want to study the behavior of a system when it is prepared and started in a state, which is the fixed point of the same family but of a slightly smaller region. A reasonable guess would be that frustration freeness implies that the evolution should be localized "around the boundary," and that for short times, nothing at all would happen in the "bulk" (where the state is left invariant by the local interaction terms because of frustration freeness). This intuition is formalized in the following lemma.

Lemma 12. Let $\mathcal{L}=(\mathcal{M}, \mathcal{B})$ be a uniform family of Lindbladians, satisfying frustration freeness. Let $A \subset \Gamma$ be a finite region and fix a positive natural number $m$. Let $B=A(m+1), R=$ $A(m+1) \backslash A(m)$, and $\rho_{\infty}^{m}$ a fixed point of $T_{t}^{\bar{A}(m)}$ and $\tau$ an arbitrary state on $R$ (see Figure 1 ),

$$
\left\|\left(T_{t}^{\bar{B}}-T_{t}^{\bar{B} \backslash A}\right)\left(\rho_{\infty}^{m} \otimes \tau\right)\right\|_{1} \leq \operatorname{poly}(m) v^{-1}(m)\left[e^{v t}-1+t\right],
$$

where $T_{t}^{\bar{B} \backslash A}$ denotes the evolution generated by $\mathcal{L}^{\bar{B} \backslash A}=\sum_{Z \subset B \backslash A} \mathcal{M}_{Z}+\sum_{d \leq m+1} \mathcal{B}_{d}^{\partial B}$.

In order to prove such result, we will first prove a Lieb-Robinson-type of lemma. Denote by $\rho(t)=T_{t}^{\bar{B}}\left(\rho_{\infty}^{m} \otimes \tau\right)$. For each $X \subset B$, denote $\mathbb{L}_{X}$ the algebra generated by $\left\{\mathcal{M}_{Z} \mid Z \subset X\right\}$, which is the set of interaction terms of $\mathcal{L}^{B}$ whose support is contained in $X$.

Lemma 13. Under the same assumptions of Lemma 12, for each $K \in \mathbb{L}_{X}$, the following "LiebRobinson-like" bound holds for some positive $v$,

$$
\|K(\rho(t))\|_{1} \leq \operatorname{poly}(m)|X|\|K\|_{\diamond}\left(e^{v t}+t-1\right) v^{-1}(\operatorname{dist}(X, R)) .
$$




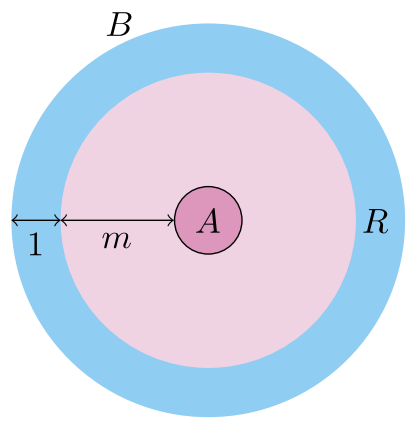

FIG. 1. The construction of the sets $B$ and $R$.

Proof. Denote $C(Z, t)=\sup _{T \in \mathbb{L}_{Z}} \frac{\|T(\rho(t))\|_{1}}{\|T\|_{\diamond}}$. Frustration-freeness implies that $C(Z, 0)$ is 0 if $Z \cap$ $R=\emptyset\left(\right.$ since $\left.\operatorname{Tr}_{R} \rho(0)=\rho_{\infty}^{m}\right)$, while it is bounded by 1 otherwise. Moreover, let $\Delta(r)=\sum_{d \geq r}\left\|\mathcal{B}_{d}^{\partial B}\right\|_{\diamond}$ and for each $Z \subset B$, let $\delta(Z)=\Delta(\operatorname{dist}(Z, R))$. Assumption (A2) implies that $\sup _{r} v(r) \Delta(r) \leq$ $\operatorname{poly}(m)$.

We are now going to replicate the proof technique of Lieb-Robinson bounds: denote $\tilde{\mathcal{L}}^{X}=$ $\mathcal{L}^{\bar{B}}-\mathcal{L}^{\bar{B} \backslash X}$, and notice that, since they have disjoint support, $\left[K, \mathcal{L}^{\bar{B} \backslash X}\right]=0$. Then,

$$
\frac{\mathrm{d}}{\mathrm{d} t} K(\rho(t))=K \mathcal{L}^{\bar{B}} \rho(t)=\mathcal{L}^{\bar{B} \backslash X} K(\rho(t))+K \tilde{\mathcal{L}}^{X}(\rho(t)),
$$

and consequently,

$$
K(\rho(t))=e^{t \mathcal{L}^{\bar{B} \backslash X}} K(\rho(0))+\int_{0}^{t} e^{(t-s) \mathcal{L}^{\bar{B} \backslash X}} K \tilde{\mathcal{L}}^{X} \rho(s) \mathrm{d} s .
$$

By taking norms

$$
\|K(\rho(t))\|_{1} \leq\|K(\rho(0))\|_{1}+\|K\|_{\diamond} \int_{0}^{t}\left\|\tilde{\mathcal{L}}^{X} \rho(s)\right\|_{1} \mathrm{~d} s,
$$

and thus,

$$
\begin{aligned}
C(X, t) \leq C(X, 0)+\sum_{Z \cap X \neq \emptyset} \int_{0}^{t}\left\|\mathcal{M}_{Z}\right\|_{\diamond} C(Z, s) \mathrm{d} s+\sum_{d>m+1} \int_{0}^{t}\left\|\mathcal{B}_{d}^{\partial B}\right\|_{\diamond} \mathrm{d} s & \\
& \leq C(X, 0)+\delta(X) t+\sum_{Z \cap X \neq \emptyset}\left\|\mathcal{M}_{Z}\right\|_{\diamond} \int_{0}^{t} C(Z, s) \mathrm{d} s .
\end{aligned}
$$

By recursively applying Equation (15), we obtain that

$$
C(X, t) \leq \sum_{n=0}^{\infty}\left[a_{n}+b_{n}\right] \frac{t^{n}}{n !},
$$

where $a_{0}=C(X, 0)$,

$$
a_{n}=\sum_{Z_{1} \cap X \neq 0} \ldots \sum_{Z_{n} \cap Z_{n-1} \neq 0}\left\|\mathcal{M}_{Z_{1}}\right\| \ldots\left\|\mathcal{M}_{Z_{n}}\right\| C\left(Z_{n}, 0\right)
$$

$b_{0}=0, b_{1}=\delta(X)$, and

$$
b_{n+1}=\sum_{Z_{1} \cap X \neq 0} \ldots \sum_{Z_{n} \cap Z_{n-1} \neq 0}\left\|\mathcal{M}_{Z_{1}}\right\| \ldots\left\|\mathcal{M}_{Z_{n}}\right\| \delta\left(Z_{n}\right) .
$$

Let us bound the two coefficients independently. The coefficients $a_{n}$ are treated in the same way as is done in the standard proof of Lieb-Robinson bounds: ${ }^{45}$ recalling that $C(Z, 0)$ is zero unless $Z \cap R \neq \emptyset$, we have that

$$
a_{n}=\sum_{Z_{1} \cap X \neq 0} \ldots \sum_{\substack{Z_{n} \cap Z_{n-1} \neq 0 \\ Z_{n} \cap R \neq 0}}\left\|\mathcal{M}_{Z_{1}}\right\| \ldots\left\|\mathcal{M}_{Z_{n}}\right\| .
$$


We have that $a_{1}$ is bounded by using Assumption (A1),

$$
a_{1} \leq \sum_{i \in X} \sum_{Z_{1} \ni i Z_{1} \cap R \neq 0}\left\|\mathcal{M}_{Z_{1}}\right\| \leq \sum_{i \in X} v^{-1}(\operatorname{dist}(i, R)) \sum_{\substack{Z_{1} \ni i \\ Z_{1} \cap R \neq 0}}\left\|\mathcal{M}_{Z_{1}}\right\| v\left(\operatorname{diam}\left(Z_{1}\right)\right) \leq v \sum_{i \in X} v^{-1}(\operatorname{dist}(i, R)) .
$$

Similarly, we bound $a_{2}$ as follows:

$$
a_{2} \leq \sum_{i \in X} \sum_{Z_{1} \ni i} \sum_{j \in Z_{2}} \sum_{\substack{Z_{2} \ni j \\ Z \cap Y \neq 0}}\left\|\mathcal{M}_{Z_{1}}\right\|\left\|\mathcal{M}_{Z_{2}}\right\| .
$$

We now use the fact that $v(\operatorname{dist}(i, j)) v(\operatorname{dist}(j, R)) \geq v(\operatorname{dist}(i, R))$, and thus,

$$
\begin{gathered}
a_{2} \leq \sum_{i \in X} v^{-1}(\operatorname{dist}(i, R)) \sum_{Z_{1} \ni i}\left\|\mathcal{M}_{Z_{1}}\right\| \sum_{j \in Z_{2}} v(\operatorname{dist}(i, j)) \sum_{\substack{Z_{2} \ni j \\
Z \cap Y \neq 0}} v(\operatorname{dist}(j, Y))\left\|\mathcal{M}_{Z_{2}}\right\| \\
\leq \sum_{i \in X} v^{-1}(\operatorname{dist}(i, R)) \sum_{Z_{1} \ni i}\left\|\mathcal{M}_{Z_{1}}\right\| v\left(\operatorname{diam}\left(Z_{1}\right)\right) \sum_{j \in Z_{1}} \sum_{\substack{Z_{2} \ni j \\
Z \cap Y \neq 0}} v\left(\operatorname{diam}\left(Z_{2}\right)\right)\left\|\mathcal{M}_{Z_{2}}\right\| \\
\leq v \sum_{i \in X} v^{-1}(\operatorname{dist}(i, R)) \sum_{Z_{1} \ni i}\left\|\mathcal{M}_{Z_{1}}\right\| v\left(\operatorname{diam}\left(Z_{1}\right)\right)\left|Z_{1}\right| \leq v^{2} \sum_{i \in X} v^{-1}(\operatorname{dist}(i, R)) .
\end{gathered}
$$

Proceeding in a similar way, we can bound $a_{n}$ by $v^{n} \sum_{i \in X} v^{-1}(\operatorname{dist}(i, R))$. Let us now turn our attention to $b_{n}$. Let $Z_{1} \cap X \neq \emptyset$. Then, for all $u$ in $Z_{1}$, it holds that $\operatorname{dist}(X, R) \leq \operatorname{dist}(u, X)+$ $\operatorname{dist}(u, R) \leq \operatorname{diam} Z_{1}+\operatorname{dist}(u, R)$. In particular, this holds for $y_{Z_{1}} \in Z_{1}$ such that $\operatorname{dist}\left(y_{Z_{1}}, R\right)=$ $\operatorname{dist}\left(Z_{1}, R\right)$. Therefore, we have that $1 \leq v\left(\operatorname{diam} Z_{1}\right) v\left(\operatorname{dist}\left(Z_{1}, R\right)\right) v^{-1}(\operatorname{dist}(X, R))$. We can use the previous inequality to bound $b_{2}$ as follow:

$$
\begin{array}{r}
b_{2}=\sum_{Z_{1} \cap X \neq \emptyset}\left\|M_{Z_{1}}\right\| \delta\left(Z_{1}\right) \leq v^{-1}(\operatorname{dist}(X, R)) \sum_{x \in X} \sum_{Z_{1} \ni x}\left\|M_{Z_{1}}\right\| v\left(\operatorname{diam} Z_{1}\right) \Delta\left(\operatorname{dist}\left(Z_{1}, R\right)\right) v\left(\operatorname{dist}\left(Z_{1}, R\right)\right) \\
\leq v \operatorname{poly}(m)|X| v^{-1}(\operatorname{dist}(X, R)) .
\end{array}
$$

For $b_{3}$, we reason similarly as follows:

$$
\begin{aligned}
& b_{3}=\sum_{Z_{1} \cap X \neq \emptyset} \sum_{Z_{2} \cap Z_{1} \neq \emptyset}\left\|M_{Z_{1}}\right\|\left\|M_{Z_{2}}\right\| \delta\left(Z_{2}\right) \\
& \leq \sum_{Z_{1} \cap X \neq \emptyset} \frac{\left\|M_{Z_{1}}\right\|}{v\left(\operatorname{dist}\left(Z_{1}, R\right)\right)} \sum_{z \in Z_{1}} \sum_{Z_{2} \ni Z}\left\|M_{Z_{2}}\right\| v\left(\operatorname{diam} Z_{2}\right) \Delta\left(\operatorname{dist}\left(Z_{2}, R\right)\right) v\left(\operatorname{dist}\left(Z_{2}, R\right)\right) \\
& \leq v \operatorname{poly}(m) \sum_{Z_{1} \cap X \neq \emptyset}\left\|M_{Z_{1}}\right\| v^{-1}\left(\operatorname{dist}\left(Z_{1}, R\right)\right)\left|Z_{1}\right| \leq v \operatorname{poly}(m) v^{-1}(\operatorname{dist}(X, R)) \sum_{x \in X} \sum_{Z_{1} \ni x}\left\|M_{Z_{1}}\right\|\left|Z_{1}\right| v\left(\operatorname{diam} Z_{1}\right) \\
& \leq v^{2} \operatorname{poly}(m) v^{-1}(\operatorname{dist}(X, R))|X| .
\end{aligned}
$$

Following the same argument, we can thus bound the general term $b_{n+1}$ by

$$
v^{n} \operatorname{poly}(m)|X| v^{-1}(\operatorname{dist}(X, R)) .
$$

We can then bound

$$
\sum_{n} a_{n} \frac{t^{n}}{n !} \leq\left(e^{v t}-1\right) \sum_{i \in X} v^{-1}(\operatorname{dist}(i, R)) \leq\left(e^{v t}-1\right)|X| v^{-1}(\operatorname{dist}(X, R))
$$

and

$$
\sum_{n} b_{n} \frac{t^{n}}{n !} \leq \operatorname{poly}(m) v^{-1}\left(e^{v t}-1\right)|X| v^{-1}(\operatorname{dist}(X, R))+t\left[\delta(X)-\operatorname{poly}(m)|X| v^{-1}(\operatorname{dist}(X, R))\right] .
$$

Note that, because of Assumption (A2), the last term in the r.h.s. can be bounded as

$$
\delta(X)-\operatorname{poly}(m)|X| v^{-1}(\operatorname{dist}(X, R)) \leq \operatorname{poly}(m)|X| v^{-1}(\operatorname{dist}(X, R)) .
$$

This concludes the proof. 
We can now prove Equation (13).

Proof. Applying Duhamel's formula ${ }^{46}$ we have that

$$
\left(T_{t}^{\bar{B}}-T_{t}^{\bar{B} \backslash A}\right)\left(\rho_{\infty}^{m} \otimes \tau\right)=\int_{0}^{t} T_{t-s}^{\bar{B} \backslash A} \tilde{\mathcal{L}}^{A} \rho(s) \mathrm{d} s,
$$

and therefore,

$$
\left\|\left(T_{t}^{\bar{B}}-T_{t}^{\bar{B} \backslash A}\right)\left(\rho_{\infty}^{m} \otimes \tau\right)\right\|_{1} \leq \sum_{Z \cap A \neq \emptyset} \int_{0}^{t}\left\|\mathcal{M}_{Z} \rho(s)\right\|_{1} \mathrm{~d} s+\sum_{d>m+1}\left\|B_{d}^{\partial B}\right\|_{\diamond} t .
$$

The second term on the r.h.s. is bounded by $t \operatorname{poly}(m) v^{-1}(m)$. Let us focus on the first term on the r.h.s. If $Z \subset A(m)$, we can bound the r.h.s with Equation (14). In particular, we have the following:

$$
\sum_{\substack{Z \cap A \neq \emptyset \\ Z \subset A(m)}}\left\|\mathcal{M}_{Z} \rho(s)\right\|_{1} \leq \operatorname{poly}(m)\left(e^{v s}+t-1\right) \sum_{\substack{Z \cap A \neq \emptyset \\ Z \subset A(m)}}\left\|\mathcal{M}_{Z}\right\|_{\diamond}|Z| v^{-1}(\operatorname{dist}(Z, R)) .
$$

Observe that since $Z \cap A \neq \emptyset$, it holds that $\operatorname{dist}(Z, R)+\operatorname{diam} Z \geq m$, and therefore,

$$
\sum_{\substack{Z \cap A \neq \emptyset \\ Z \subset A(m)}}\left\|\mathcal{M}_{Z}\right\|_{\diamond}|Z| v^{-1}(\operatorname{dist}(Z, R)) \leq v^{-1}(m) \sum_{\substack{Z \cap A \neq \emptyset \\ Z \subset A(m)}}\left\|\mathcal{M}_{Z}\right\|_{\diamond}|Z| v(\operatorname{diam} Z) \leq v^{-1}(m) v,
$$

where we have used the Lieb-Robinson assumption.

If $Z \nsubseteq A(m)$, then it must hold that $Z \cap R \neq \emptyset$. Then, we showed in the previous lemma that

$$
\sum_{\substack{Z \cap A \neq \emptyset \\ Z \cap R \neq \emptyset}}\left\|\mathcal{M}_{Z}\right\|_{1} \leq v|A| v^{-1}(\operatorname{dist}(A, R))=v|A| v^{-1}(m) .
$$

Putting it all together, we have that

$$
\left\|\left(T_{t}^{\bar{B}}-T_{t}^{\bar{B} \backslash A}\right)\left(\rho_{\infty}^{m} \otimes \tau\right)\right\|_{1} \leq \operatorname{poly}(m) v^{-1}(m)\left[e^{v t}-1+t\right] .
$$

\section{DECAY OF CORRELATIONS}

In this section, we show that as a straightforward consequence of Lemma 11, the hypotheses on $\mathcal{L}$ imply that its fixed points have a particular character: they have fast decay of correlations, meaning that the correlations between two spatially separated regions are fast-decaying in distance. How fast this decay is given by the decaying function $\Delta_{0}$ defined in Lemma 11 .

Theorem 14. Under the same assumption as in Lemma 11, fix two regions $A$ and $B \subset \Lambda$, let $d_{A B}>0$ be the distance between them. Then, we have that

$$
T(A: B) \leq 3(|A|+|B|)^{\delta} \Delta_{0}\left(\frac{d_{A B}}{2}\right),
$$

where the correlations are calculated with respect to $\rho_{\infty}$, and $\delta$ and $\Delta_{0}$ are defined in Lemma 11.

Proof. Let $C=A \cup B$, and denote by $\rho_{A B}$ the reduced density matrix of $\rho_{\infty}$ over $C$, and by $\rho_{A}$ and $\rho_{B}$ the reduced state on $A$ and $B$, respectively.

Consider $\rho_{\infty}^{s}$ the unique fix point of $T_{t}^{\overline{C(s)}}$ and denote by $\rho_{A}^{s}$ and $\rho_{B}^{s}$ its reduced density matrices over $A$ and $B$, respectively. If $s \leq \frac{d_{A B}}{2}$, then $C(s)$ has two disjoint components corresponding to $A(s)$ and $B(s)$, and thus, $\rho_{\infty}^{s}$ decomposes as a tensor product over such bipartition, and its reduced density matrix over $C$ is given by $\rho_{A}^{s} \otimes \rho_{B}^{s}$.

By Lemma 11, we have that for any observable $O_{C}$ with operator norm equal to 1 and supported on $C$,

$$
\left|\operatorname{Tr} O_{C}\left(\rho_{\infty}-\rho_{\infty}^{s}\right)\right| \leq|C|^{\delta} \Delta_{0}(s)
$$


This implies that

$$
\left\|\rho_{A B}-\rho_{A}^{s} \otimes \rho_{B}^{s}\right\|_{1} \leq|C|^{\delta} \Delta_{0}(s) .
$$

Since the trace norm does not increases under the partial trace, then

$$
\left\|\rho_{A}-\rho_{A}^{s}\right\|_{1} \leq|C|^{\delta} \Delta_{0}(s),
$$

and the same holds for $B$. This in turn implies that

$$
\left\|\rho_{A} \otimes \rho_{B}-\rho_{A}^{s} \otimes \rho_{B}^{s}\right\|_{1} \leq 2|C|^{\delta} \Delta_{0}(s),
$$

and by applying the triangle inequality, we obtain the desired result.

Remark 15. By Equation (4), we have that the mutual information $I(A: B)$ decays with $\operatorname{dist}(A, B)$ at essentially the same rate as $T(A: B)$.

Note that the dependence on $|A|$ and $|B|$ of the bound in Equation (16), which is harmless when $A$ and $B$ are finite regions - as in the case of two-point correlation functions - becomes significant when one of the two regions is proportional to the system size. In Ref. 19, the authors defined a correlation length $\xi$ for the mutual information, as the minimal length such that for all $L \geq \xi$, it holds that

$$
I\left(b_{x}(R-L): b_{x}(R)^{c}\right) \leq \frac{1}{2} I\left(b_{x}(R): b_{x}(R)^{c}\right), \quad \forall x, \forall R \geq 0 .
$$

From this property, they are able to derive an area law for the mutual information of the type

$$
I\left(A: A^{c}\right) \leq 4|\partial A| \xi \text {. }
$$

If we were to use (16) to determine a correlation length $\xi$, we would obtain a value for $\xi$ which depends on the system size and thus obtain a bound comparable to Equation (1) obtained in Ref. 31 . In Sec. V, we want to show that it is possible to greatly improve this bound at the cost of adding some extra hypotheses on the evolution.

\section{AREA LAW FOR MUTUAL INFORMATION}

Until now, we have avoided as much as possible to put restrictions on the function $v(r)$ appearing in Assumptions (A1) and (A2), and we have simply required it to be fast enough for Lieb-Robinson bounds to hold. Indeed, in most of the bounds obtained in the previous results, it appears $v(r)$ (or $\Delta_{0}(r)$, which depends on $v(r)$ ), so that weaker assumptions will simply lead to weaker bounds.

This gets more complicated when it gets to prove the area law bounds, since we are actually interested in pinning down the case in which the bound on mutual information takes the form of $|\partial A| \log |A|$, an area law with a logarithmic correction. As will be clear in the proof, the logarithmic correction depends on $v(r)$ (and consequently $\Delta_{0}(r)$ ) to be exponential. Slower rates will still lead to a bound on the mutual information, but where the logarithmic correction is replaced by a super-logarithmic correction. The resulting bound can hardly be called an area law, a sub-volume law would be more correct. We will avoid such generalization, as they make the proof unnecessarily complicated and focus on the more interesting case of exponentially decaying interactions, for which we can prove a proper area law with logarithmic correction.

\section{A. The pure fixed point case}

In this section, let us suppose that each $T_{t}^{\bar{\Lambda}}$ has a unique fixed point, and moreover, that this fixed point is pure. This setting is of particular interest in view of dissipative state engineering, ${ }^{15,16}$ since ideally one would like to be able to create pure states (at least before noise and errors are taken into account).

Let us denote by $\left|\phi_{\Lambda}\right\rangle$ the pure fixed point of $T_{t}^{\bar{\Lambda}}$. 
Proposition 16. Let $\mathcal{L}=\{\mathcal{M}, \mathcal{B}\}$ be a uniform family of dissipative evolutions, satisfying rapid mixing and having a unique pure fixed point $\left|\phi_{\Lambda}\right\rangle$ for each $\Lambda$. Fix $A \subset \Lambda$, and let $\rho_{A}$ denote the reduced density matrix of $\left|\phi_{\Lambda}\right\rangle$ on $A$. Then, it holds that

$$
S\left(\rho_{A}\right) \leq c \log |A| \cdot|\partial A|,
$$

for some constant $c>0$.

Proof. Let $\ell \geq 0$, to be determined later, and denote by $\sigma$ the reduced density matrix of $\left|\phi_{A(\ell)}\right\rangle$ on $A$. Then, we have trivially that

$$
S(\sigma) \leq \log _{2} \operatorname{dim} \mathcal{H}_{A(\ell) \backslash A} \leq c_{0} \ell|\partial A|,
$$

for some positive constant $c_{0}$. On the other hand, by Lemma 11, we have that $d:=\left\|\rho_{A}-\sigma\right\|_{1} \leq$ $|A|^{\delta} \Delta_{0}(\ell)$, and thus by Equation (2),

$$
S\left(\rho_{A}\right) \leq S(\sigma)+\left|S(\sigma)-S\left(\rho_{A}\right)\right| \leq c_{0} \ell|\partial A|+2 d|A|+2 h_{b}(d) \leq c_{0} \ell|\partial A|+2|A|^{\delta+1} \Delta_{0}(\ell)+2 h_{b}(d) .
$$

Fix an $\varepsilon$ such that $0<\varepsilon<1 / 2$ and let us choose $\ell$ such that $2|A|^{\delta+1} \Delta_{0}(\ell) \leq \varepsilon$. This implies that $\ell$ scales as $\log |A|$, and thus, $S\left(\rho_{A}\right) \leq c_{1} \log |A| \cdot|\partial A|+\varepsilon+2 h_{b}(\varepsilon / 2|A|)$, for some positive $c_{1}$. By taking $c \geq c_{1}$, we can absorb the terms depending on $\varepsilon$ in the other one and obtain the claimed estimate $S\left(\rho_{A}\right) \leq c \log |A| \cdot|\partial A|$.

While of interest, the case of a pure fixed point is a very specific one. Therefore, we want to give results applicable in the generic case of a mixed fixed point. To obtain such results, we will need to make an additional assumption, namely, that the system is frustration free.

\section{B. The frustration-free case}

Theorem 17 (Area law for mutual information). Let $\mathcal{L}=\{\mathcal{M}, \mathcal{B}\}$ be a uniform family of dissipative evolutions, satisfying rapid mixing frustration freeness and having a unique pure fixed point. Let $\rho_{\infty}$ be the fixed point of $\mathcal{L}^{\bar{\Lambda}}$ for some $\Lambda=b_{u}(L) \subset \Gamma$. Then, we have that

$$
I\left(A: A^{c}\right)_{\rho_{\infty}} \leq c|\partial A| \log |A|,
$$

for some positive $c$ independent of the system-size.

Proof. For each $n \geq 0$, let $\rho_{\infty}^{n}$ be the fixed point of $T_{t}^{\bar{A}(n)}$. Fix a positive $n_{0}$ to be determined later. Then, it holds that

$$
I\left(A: A^{c}\right)_{\rho_{\infty}}=I\left(A: A^{c}\right) \rho_{\infty}^{n_{0}}+\sum_{n=n_{0}}^{L-1}\left[I\left(A: A^{c}\right) \rho_{\infty}^{n+1}-I\left(A: A^{c}\right) \rho_{\infty}^{n}\right] .
$$

We want to show that it is possible to choose $n_{0}$ in such a way that $I\left(A: A^{c}\right)_{\rho_{\infty}^{n_{0}}} \leq c|\partial A| \log |A|$ and the sum in the r.h.s. is arbitrarily small.

For each $n \geq 0$, we have that by rapid mixing (11),

$$
\left\|\rho_{\infty}^{n+1}-T_{t}^{\bar{A}(n+1)}\left(\rho_{\infty}^{n} \otimes \tau\right)\right\|_{1} \leq|A| \phi_{1}(n) e^{-\gamma t},
$$

where $\phi_{1}(n)$ is a polynomial in $n$. On the other hand, Equation (13) implies that

$$
\left\|T_{t}^{\bar{A}(n+1)}\left(\rho_{\infty}^{n} \otimes \tau\right)-T_{t}^{\bar{A}(n+1) \backslash A}\left(\rho_{\infty}^{n} \otimes \tau\right)\right\|_{1} \leq|A| \phi_{2}(n) v^{-1}(n)\left[e^{v t}+t-1\right],
$$

and $\phi_{2}(n)$ is polynomial in $n$. Let us choose $t_{n}$ such that

$$
\varepsilon_{n}:=\phi_{1}(n) e^{-\gamma t_{n}}+\phi_{2}(n) v^{-1}(n)\left[e^{v t_{n}}+t_{n}-1\right]
$$

is exponentially decaying in $n$. This can be done by taking $t_{n}$ which scales proportionally to $\frac{1}{v+\gamma} \log \left(v(n) \frac{\phi_{1}(n)}{\phi_{2}(n)}\right)$, which is essentially linear if $v(n)$ grows exponentially. We can put the two bounds together using the triangle inequality in such a way that

$$
\left\|\rho_{\infty}^{n+1}-T_{t_{n}}^{\bar{A}(n+1) \backslash A}\left(\rho_{\infty}^{n} \otimes \tau\right)\right\|_{1} \leq|A| \varepsilon_{n} .
$$


Observe that since $T_{t}^{\bar{A}(n+1) \backslash A}$ does not act on $A, I\left(A: A^{c}\right)_{T_{t}^{\bar{A}(n+1) \backslash A}\left(\rho_{\infty}^{n} \otimes \tau\right)} \leq I\left(A: A^{c}\right)_{\rho_{\infty}^{n}}$.

Let us assume that $n_{0}$ is big enough so that $\varepsilon_{n}|A|^{2} \leq 2^{-n}$ for all $n \geq n_{0}$, we can then apply inequality (4) and obtain

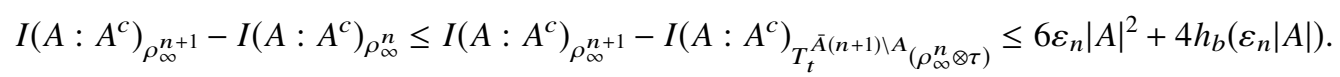

Then,

$$
I\left(A: A^{c}\right)_{\rho_{\infty}} \leq I\left(A: A^{c}\right)_{\rho_{\infty}}^{n_{0}}+6 \sum_{n=n_{0}}^{L-1} 2^{-n}+4 \sum_{n=n_{0}}^{L-1} h_{b}\left(\varepsilon_{n}|A|\right) .
$$

Observe that if $0 \leq x \leq 1 / e$, then $(x-1) \log _{2}(1-x) \leq-x \log _{2} x$, and the latter is an increasing function in that interval. Therefore,

$$
h_{b}\left(\varepsilon_{n}|A|\right) \leq-2 \varepsilon_{n}|A| \log _{2}\left(\varepsilon_{n}|A|\right) \leq \frac{2^{-n+1}}{|A|}\left(n+\log _{2}|A|\right) .
$$

Therefore, $\sum_{n=n_{0}}^{L-1} h_{b}\left(\varepsilon_{n}|A|\right)$ is the tail of a series which is converging geometrically, and therefore, it is exponentially decaying as $n_{0}$ increases. The same is true for $\sum_{n=n_{0}}^{L-1} \lambda^{n}$, so that both of them can be made smaller than $I\left(A: A^{c}\right) \rho_{\infty}^{n_{0}}$.

By taking $n_{0}$ proportional to $\log |A|$, we can bound $I\left(A: A^{c}\right)_{\rho_{\infty}}^{n_{0}}$ by the logarithm of $\operatorname{dim} \mathcal{H}_{A^{c}}$, which is proportional to $\left|A\left(n_{0}\right) \backslash A\right|$, and therefore,

$$
I\left(A: A^{c}\right)_{\rho_{\infty}}^{n_{0}} \leq c|\partial A| \log |A| .
$$

In conclusion, we have bounded the r.h.s. of (18) by $c|\partial A| \log |A|$, and this concludes the proof.

\section{ACKNOWLEDGMENTS}

F.G.S.L.B. is supported by EPSRC. T.S.C. is supported by the Royal Society. A.L. is supported by MINECO FPI fellowship No. BES-2012-052404. A.L. and D.P.-G. acknowledge support from MINECO (Grant No. MTM2011-26912), Comunidad de Madrid (Grant No. QUITEMAD+CM, Ref. No. S2013/ICE-2801) and the European CHIST-ERA project CQC (funded partially by MINECO under Grant No. PRI-PIMCHI-2011-1071). This work was made possible through the support of grant \#48322 from the John Templeton Foundation. The opinions expressed in this publication are those of the authors and do not necessarily reflect the views of the John Templeton Foundation. This project has received funding from the European Research Council (ERC) under the European Union's Horizon 2020 research and innovation programme (Grant Agreement No. 648913). S.M. acknowledges funding provided by IQIM, a NSF Physics Frontiers Center with support of the Gordon and Betty Moore Foundation through Grant No. GBMF1250, and AFOSR Grant No. FA8750-12-2-0308. The authors would like to thank the hospitality of the Isaac Newton Institute for Mathematical Sciences, where part of this work was carried out.

${ }^{1}$ L. Masanes, "Area law for the entropy of low-energy states," Phys. Rev. A 80, 052104 (2009).

${ }^{2}$ B. Swingle and J. McGreevy, "Area law for gapless states from local entanglement thermodynamics," e-print arXiv:1505. 07106v1 [cond-mat.str-el] (2015).

${ }^{3}$ Some authors call such situation a violation of the area law, while others speak of an area law with a logarithmic correction. We will use the second terminology, but we will use the term sub-volume law for any faster growth rate of entanglement that is slower than the volume of the region.

${ }^{4}$ M. B. Hastings, "An area law for one-dimensional quantum systems," J. Stat. Mech.: Theory Exp. 2007, P08024; e-print arXiv:0705.2024 [quant-ph].

${ }^{5}$ I. Arad, A. Kitaev, Z. Landau, and U. Vazirani, "An area law and sub-exponential algorithm for 1D systems," e-print arXiv:1301.1162 [quant-ph] (2013)

${ }^{6}$ I. Arad, Z. Landau, and U. Vazirani, “Improved one-dimensional area law for frustration-free systems,” Phys. Rev. B 85, 195145 (2012).

${ }^{7}$ F. G. S. L. Brandao and M. Horodecki, "An area law for entanglement from exponential decay of correlations," Nat. Phys. 9, 721-726 (2013).

${ }^{8}$ F. G. S. L. Brandao and M. Horodecki, "Exponential decay of correlations implies area law,” Commun. Math. Phys. 333, 761 (2015). 
${ }_{9}^{9}$ M. B. Plenio, J. Eisert, J. Dreißig, and M. Cramer, "Entropy, entanglement, and area: Analytical results for harmonic lattice systems,” Phys. Rev. Lett. 94, 060503 (2005).

${ }^{10}$ S. Michalakis and J. Pytel, "Stability of frustration-free Hamiltonians," Commun. Math. Phys. 322(2), 277-302 (2013); e-print arXiv:1109.1588 [quant-ph] (2011).

${ }^{11}$ S. Michalakis, "Stability of the area law for the entropy of entanglement," e-print arXiv:1206.6900v2 [quant-ph] (2012).

${ }^{12}$ M. Mariën, K. M. R. Audenaert, K. V. Acoleyen, and F. Verstraete, "Entanglement rates and the stability of the area law for the entanglement entropy," e-print arXiv:1411.0680v1 [math-ph] (2014).

${ }^{13}$ M. M. Wolf, "Violation of the entropic area law for fermions," Phys. Rev. Lett. 96, 010404 (2006).

${ }^{14}$ D. Gioev and I. Klich, "Entanglement entropy of fermions in any dimension and the Widom conjecture," Phys. Rev. Lett. 96, 100503 (2006).

${ }^{15}$ B. Kraus, H. P. Büchler, S. Diehl, A. Kantian, A. Micheli, and P. Zoller, "Preparation of entangled states by quantum Markov processes," Phys. Rev. A 78, 042307 (2008); e-print arXiv:0803.1463 [quant-ph].

${ }^{16}$ F. Verstraete, M. M. Wolf, and J. I. Cirac, "Quantum computation and quantum-state engineering driven by dissipation," Nat. Phys. 5, 633-636 (2009); e-print arXiv:0803.1447 [quant-ph].

${ }^{17}$ H. Krauter, C. A. Muschik, K. Jensen, W. Wasilewski, J. M. Petersen, J. I. Cirac, and E. S. Polzik, "Entanglement generated by dissipation and steady state entanglement of two macroscopic objects," Phys. Rev. Lett. 107, 080503 (2011).

${ }^{18}$ J. T. Barreiro, P. Schindler, O. Gühne, T. Monz, M. Chwalla, C. F. Roos, M. Hennrich, and R. Blatt, "Experimental multiparticle entanglement dynamics induced by decoherence,” Nat. Phys. 6, 943-946 (2010); e-print arXiv:1005.1965 [quant-ph]

${ }^{19}$ M. M. Wolf, F. Verstraete, M. B. Hastings, and J. I. Cirac, "Area laws in quantum systems: Mutual information and correlations," Phys. Rev. Lett. 100, 070502 (2008).

${ }^{20}$ C. H. Bennett, D. P. DiVincenzo, J. A. Smolin, and W. K. Wootters, "Mixed-state entanglement and quantum error correction,” Phys. Rev. A 54, 3824 (1996).

${ }^{21}$ O. Szehr, D. Reeb, and M. M. Wolf, "Spectral convergence bounds for classical and quantum Markov processes," Comm. Math. Phys. 333(2), 565-595 (2015); e-print arXiv:1301.4827 [quant-ph] (2013).

22 T. S. Cubitt, A. Lucia, S. Michalakis, and D. Perez-Garcia, "Stability of local quantum dissipative systems," Comm. Math. Phys. 337(3), 1275-1315 (2015); e-print arXiv:1303.4744 [quant-ph] (2013).

${ }^{23}$ M. J. Kastoryano, D. Reeb, and M. M. Wolf, "A cutoff phenomenon for quantum Markov chains," J. Phys. A 45, 075307, 16 (2012); e-print arXiv:1111.2123 [quant-ph].

${ }^{24}$ F. Martinelli, E. Olivieri, and R. H. Schonmann, "For 2-D lattice spin systems weak mixing implies strong mixing," Commun Math. Phys. 165, 33-47 (1994).

${ }^{25}$ E. Lubetzky and A. Sly, "Cutoff for the Ising model on the lattice," Invent. Math. 191, 719-755 (2013); e-print arXiv:0909. 4320 [math.PR].

${ }^{26}$ L. Gross, "Logarithmic Sobolev inequalities,” Am. J. Math. 97, 1061-1083 (1975).

${ }^{27}$ L. Gross, "Logarithmic Sobolev inequalities and contractivity properties of semigroups," in Dirichlet Forms (Varenna, 1992), Lecture Notes in Mathematics Vol. 1563 (Springer, Berlin, 1993), pp. 54-88.

${ }^{28}$ L. Gross, "Hypercontractivity, logarithmic Sobolev inequalities, and applications: A survey of surveys," in Diffusion, Quantum Theory, and Radically Elementary Mathematics, Mathematics Notes Vol. 47 (Princeton University Press, Princeton, NJ, 2006), pp. 45-73.

${ }^{29}$ M. J. Kastoryano and K. Temme, "Quantum logarithmic Sobolev inequalities and rapid mixing,” J. Math. Phys. 54, 052202 (2013); e-print arXiv:1207.3261 [quant-ph] (2012).

${ }^{30} \mathrm{~N}$. Yoshida, "The equivalence of the log-Sobolev inequality and a mixing condition for unbounded spin systems on the lattice," Ann. Inst. Henri Poincare, Sect. B: Probab. Stat. 37, 223-243 (2001).

${ }^{31}$ M. J. Kastoryano and J. Eisert, "Rapid mixing implies exponential decay of correlations," J. Math. Phys. 54, 102201 (2013); e-print arXiv:1303.6304 [quant-ph].

${ }^{32}$ M. A. Nielsen and I. L. Chuang, Quantum Computation and Quantum Information (Cambridge University Press, 2010).

${ }^{33}$ K. M. R. Audenaert, “A sharp continuity estimate for the von Neumann entropy,” J. Phys. A 40, 8127-8136 (2007); e-print arXiv:quant-ph/0610146.

${ }^{34}$ M. Fannes, "A continuity property of the entropy density for spin lattice systems," Commun. Math. Phys. 31, 291-294 (1973).

${ }^{35}$ R. Alicki and M. Fannes, “Continuity of quantum conditional information,” J. Phys. A 37, L55-L57 (2004); e-print arXiv: quant-ph/0312081.

${ }^{36}$ M. M. Wolf, Quantum channels \& operations. Guided tour http://www-m5.ma.tum.de/foswiki/pub/M5/Allgemeines/ MichaelWolf/QChannelLecture.pdf.

${ }^{37}$ V. Gorini, A. Kossakowski, and E. C. G. Sudarshan, "Completely positive dynamical semigroups of $N$-level systems," J. Math. Phys. 17, 821-825 (1976).

${ }^{38}$ G. Lindblad, "On the generators of quantum dynamical semigroups," Commun. Math. Phys. 48, 119-130 (1976).

${ }^{39} \mathrm{H}$. Breuer and F. Petruccione, The Theory of Open Quantum Systems (Oxford University Press, 2002).

${ }^{40}$ M. J. Kastoryano and F. G. S. L. Brandao, "Quantum Gibbs samplers: The commuting case," e-print arXiv:1409. 3435 [quant-ph] (2014).

${ }^{41}$ D. W. Robinson, "Statistical mechanics of quantum spin systems. II," Commun. Math. Phys. 7, 337-348 (1968).

${ }^{42}$ E. H. Lieb and D. W. Robinson, "The finite group velocity of quantum spin systems," Commun. Math. Phys. 28, 251-257 (1972).

${ }^{43}$ D. Poulin, "Lieb-Robinson bound and locality for general Markovian quantum dynamics," Phys. Rev. Lett. 104, 190401 (2010); e-print arXiv: 1003.3675 [quant-ph].

${ }^{44}$ B. Nachtergaele, A. Vershynina, and V. A. Zagrebnov, "Lieb-Robinson bounds and existence of the thermodynamic limit for a class of irreversible quantum dynamics," in Entropy and the Quantum II, Contemporary Mathematics Vol. 552 (American Mathematical Society, Providence, RI, 2011), pp. 161-175; e-print arXiv:1103.1122 [math-ph].

${ }^{45}$ M. B. Hastings, "Locality in quantum systems," e-print arXiv:1008.5137 [math-ph] (2010).

${ }^{46}$ T. Tao, Nonlinear Dispersive Equations: Local and Global Analysis (American Mathematical Society, 2006), Vol. 106. 